\title{
Probabilistic Constructive Interference Precoding for Imperfect CSIT
}

\author{
Gangming Lyu, Yuning You, Ang Li, Member, IEEE, Xuewen Liao and Christos Masouros, Senior Member, IEEE
}

\begin{abstract}
This paper proposes a stochastic-robust constructive interference (CI) precoding scheme for downlink multi-user MISO systems, assuming that channel state information (CSI) at the transmitter side (CSIT) is contaminated by Gaussiandistributed uncertainties. Our objective is to minimize the total transmit power under users' quality-of-service constraints: formulating CI at each user with high probabilities for a given target signal-to-noise ratio (SINR). We first analyze the probability of CI under imperfect CSIT. A series of approximations are then developed, transforming the intractable stochastic CI constraints into determined convex constraints. The non-convex stochasticrobust CI power minimization (CIPM) problem is then converted into second-order cone programming. We show that we could create tightened or relaxed approximations by changing the parameters, enabling us to find upper-bounds and lower-bounds for the original stochastic CIPM problem. The best parameter values corresponding to the tightest upper and lower bounds are also discussed and obtained. Simulation results show that the proposed methods reasonably approximate the stochastic CIPM problem. Using the given parameter values, it can guarantee the required probability of $\mathrm{CI}$ for each user under acceptable channel uncertainties and outperform the existing robust CI precoding in terms of both transmit power and feasibility rate. The small gap between the upper and lower bounds also shows that the proposed method does not cause too much performance loss.
\end{abstract}

Index Terms-MIMO, robust precoding, constructive interference, imperfect CSI, optimization

\section{INTRODUCTION}

A $\mathrm{S}$ one of the key enabling technology for multi-user multi-antenna communication systems, precoding has received extensive research interest in recent years [1], most of

Manuscript received July 14, 2020; revised December 31, 2020; accepted March 01, 2021. The associate editor coordinating the review of this paper and approving it for publication was Prof. Jingon Joung. (Corresponding author: Ang $\mathrm{Li}$ )

Copyright (c) 2015 IEEE. Personal use of this material is permitted. However, permission to use this material for any other purposes must be obtained from the IEEE by sending a request to pubs-permissions@ ieee.org.

G. Lyu, A. Li and X. Liao are with the School of Information and Communications Engineering, Xi'an Jiaotong University, Xi'an, Shaanxi 710049, China (email: gmlv@xjtu.edu.cn, ang.li.2020@xjtu.edu.cn, yeplos@mail.xjtu.edu.cn).

Y. You was with the School of Information and Communications Engineering, Xi'an Jiaotong University, Xi'an, Shaanxi 710049, China (email: yuning.you@foxmail.com).

C. Masouros is with the Department of Electronics and Electrical Engineering, University College London, London, U.K. (email: c.masouros@ucl.ak.uk).

This work is supported in part by the Science and Technology Program of Shaanxi Province under Grant No. 2019KW-007 and 2020KW-007, in part by the Fundamental Research Funds for the Central Universities under Grant xzy012020007 and xzy012019037, in party by the open research fund of National Mobile Communications Research Laboratory, Southeast University under Grant No. 2020D12. which in general have assumed perfect channel state information (CSI) at the transmitter side (CSIT). However, in practical wireless scenarios, CSIT is imperfect due to several effects such as channel estimation errors, delay, frequency offset, defective hardware components, etc. [2], [3]. Therefore, robust designs that consider these uncertainties play an indispensable part in the practical implementation of multi-user transmission. In the literature, popular robust precoding designs include the worst-case robust that guarantees the quality of service (QoS) of each user in the worst case, which applies to FDD systems with norm-bounded CSI errors (CE) [2], as well as the stochastic robust that ensure the QoS for each user with an outage probability tolerance, which applies to the TDD systems with stochastic CEs [3].

The above robust designs are studied for traditional blocklevel precoding algorithms, where the precoding matrix is applied to a block of data symbol vectors. More recently, the symbol-level constructive interference (CI) precoding, which exploits the instantaneous multi-user interference instead of suppressing it as done in traditional precoding, has received increasing research attention due to its superior performance over block-level precoding methods [4]. The main idea of CI precoding is to design the transmit signal on a symbol level such that for each user, the multi-user interference is manipulated and enforced to lie in the constructive region of the desired data symbol [5]-[7]. Early works on CI precoding have focused on the adaptation of traditional linear precoding methods by handling the interference more elaborately [8], [9]. Later, it was combined with optimization, and the concept of symbol-level precoding is proposed [5], [10]-[14]. In [10], CI is required to be strictly aligned to the desired data symbol, which is shown to be sub-optimal later [13]. In [11] a relaxed CI region is proposed for phase-shift keying (PSK) systems achieving further performance improvements and is extended to generic constellations in [14]. Due to CI precoding methods' great potential for power saving, these works have focused mainly on the CI power minimization (CIPM) problem. Recently, topics such as SINR balancing [15], symbol error rate (SER) minimization [16], CI security [17] and hardware efficient CI [18] are also studied in literature.

Most of the works on CI precoding have assumed perfect CSIT, while it is inevitable that inaccurate CSIT may cause performance degradation. Therefore, the effect of $\mathrm{CE}$ needs to be addressed for CI precoding. In [5], ellipsoid bounded CE is considered for CIPM problem in multi-user multi-input singleoutput (MU-MISO) systems, and a robust scheme is proposed which aims to guarantee the SINR for each user in the worst case. In [20], unpredictable CE caused by quantization is 
studied for CI precoding and is also captured as ellipsoid bounded uncertainties. In [21] and [22], stochastic robust CIPMs are studied for single-cell and multi-cell systems, respectively, which try to ensure the CI for each user with a given outage probability. The main challenge is to handle the intractable probabilistic CI constraints that arise in the problems. In [21], three convex approximation methods are proposed based on the idea of converting the probability of jointly distributed randoms into that of independent randoms, which does not work well due to a mathematical flaw in the transformation (see equation (18) in [21]). In [22], an approximation method is developed based on decoupling the probabilistic CI constraint of each user into two independent probabilistic constraints, which creates only a necessary condition for the original constraints and cannot guarantee users' outage requirements.

This paper proposes a new way of handling the probabilistic constraint for downlink MU-MISO systems assuming Gaussian distributed CE. The intractable stochastic robust CIPM problem is transformed into a convex problem. We show that such transformation is replacing the original feasible set with a pie slice set. By adjusting the parameter, different approximations can be realized. The best parameters for the tightened approximation and relaxed approximation are also discussed and developed, using which the lowest upper-bound and the largest lower-bound can be obtained. Simulation results show that the proposed method can reliably meet users' outage requirements under acceptable channel errors and outperform the existing outage-guaranteed robust CI schemes in terms of both transmit power and feasibility rate. Besides, the performance gaps of transmit power, outage probability, and symbol error rate between the upper and lower bound are all shown to be small, which implies that the loss caused by the proposed method is negligible compared to the optimal solution.

Notation: $\boldsymbol{I}_{K}$ is the $K \times K$ identity matrix, $\operatorname{Pr}\{\cdot\}$ denotes the probability of an event, $\mathcal{N}=\{1, \cdots, N\}, \boldsymbol{T}(\boldsymbol{x})=$ $[\operatorname{Re}(\boldsymbol{x}) ; \operatorname{Im}(\boldsymbol{x})]$ is a column vector composed by the real part $\operatorname{Re}(\boldsymbol{x})$ and the imaginary part $\operatorname{Im}(\boldsymbol{x})$ of a column vector $\boldsymbol{x}, \operatorname{erf}(z)=\frac{2}{\sqrt{\pi}} \int_{0}^{z} e^{-t^{2}} d t$ is the error function, $\succeq$ stands for component-wise greater or equal, $f^{-1}(\cdot)$ is the inverse function of $f(\cdot)$ and $\Phi$ denotes an empty set.

\section{System Model AND PRoblem Formulation}

We consider a single-cell multi-user MISO downlink transmission, where a base station (BS) equipped with $M$ transmit antennas communicates with a total number of $N$ singleantenna users. We assume that the adopted modulation method is unit-norm $\mathcal{M}$-PSK, and $d_{i} \in\{\exp (j(2 m-1) \theta)\}_{m=1}^{\mathcal{M}}$ denotes the data symbol for the $i$ th user with $\theta=\frac{\pi}{\mathcal{M}}$. To guarantee the detection performance, a minimum signal to interference noise ratio (SINR) of $\hat{\gamma}_{i}$ is required by the $i$ th user. The channel between transmitter and receivers are assumed to be block quasi-static and flat fading which remains invariant in one symbol period. Thus the received signal at the $i$ th user can be written as

$$
y_{i}=\boldsymbol{h}_{i}^{\mathrm{T}} \boldsymbol{x}+z_{i},
$$

where $\boldsymbol{x} \in \mathcal{C}^{M \times 1}$ is the transmitted signal vector from BS, $\boldsymbol{h}_{i} \in \mathcal{C}^{M \times 1}$ is the channel between BS and the $i$ th user and $z_{i} \sim \mathcal{C N}\left(0, \sigma_{z_{i}}^{2}\right)$ is the additive complex Gaussian noise. Imperfect CSIT is considered with $\boldsymbol{h}_{i}$ being modeled as

$$
\boldsymbol{h}_{i}=\boldsymbol{h}_{\mathrm{est}, i}+\boldsymbol{e}_{i}
$$

where $\boldsymbol{h}_{\text {est, } i}$ is the estimated CSI known at the transmitter and $\boldsymbol{e}_{i} \sim \mathcal{C N}\left(\mathbf{0}, \boldsymbol{\Sigma}_{\boldsymbol{e}_{i}}\right)$ stands for the channel error which is modeled as a complex Gaussian random vector with mean $\mathbf{0}$ and covariance $\boldsymbol{\Sigma}_{\boldsymbol{e}_{i}}$. Moreover, we assume $\boldsymbol{\Sigma}_{\boldsymbol{e}_{i}}=$ $\operatorname{diag}\left(\sigma_{\boldsymbol{e}_{i}, 1}^{2}, \ldots, \sigma_{\boldsymbol{e}_{i}, M}^{2}\right)$ which indicates that the channel errors for different transmit-receive antenna pairs are independent.

Our objective in this paper is to minimize the transmit power while meeting the SINR requirement of each user using CI precoding which is also known as CIPM problem [5]. Taking into consideration the imperfect CSIT, the real-valued representation of CIPM precoding can be formulated as

$$
\begin{aligned}
\mathcal{P} 1(\mathrm{CIPM}): & \min _{\tilde{\boldsymbol{x}}}\|\tilde{\boldsymbol{x}}\|^{2} \\
\text { s.t. }(\mathrm{C} 1): & \left|\left(\tilde{\boldsymbol{h}}_{i}+\tilde{\boldsymbol{e}}_{i}\right)^{\mathrm{T}} \boldsymbol{B} \tilde{\boldsymbol{x}}\right| / \tan \theta \\
& \leq\left(\tilde{\boldsymbol{h}}_{i}+\tilde{\boldsymbol{e}}_{i}\right)^{\mathrm{T}} \boldsymbol{A} \tilde{\boldsymbol{x}}-\sqrt{\hat{\gamma}_{i}} \sigma_{z_{i}}, \forall i \in \mathcal{N},
\end{aligned}
$$

where $\tilde{\boldsymbol{x}}=\boldsymbol{T}(\boldsymbol{x}), \quad \tilde{\boldsymbol{e}}_{i}=\boldsymbol{T}\left(d_{i}^{*} \boldsymbol{e}_{i}\right), \tilde{\boldsymbol{h}}_{i}=\boldsymbol{T}\left(d_{i}^{*} \boldsymbol{h}_{\mathrm{est}, i}\right)$, $\boldsymbol{A}=\left[\boldsymbol{I}_{M}, \mathbf{0} ; \mathbf{0},-\boldsymbol{I}_{M}\right], \boldsymbol{B}=\left[\mathbf{0}, \boldsymbol{I}_{M} ; \boldsymbol{I}_{M}, \mathbf{0}\right]$, and constraint C1 stands for the conditions of distance preserve CI in PSK systems [5], [23]. Let $\widetilde{\boldsymbol{\Sigma}}_{\boldsymbol{e}_{i}}=\left[\boldsymbol{\Sigma}_{\boldsymbol{e}_{i}}, \mathbf{0} ; \mathbf{0}, \boldsymbol{\Sigma}_{\boldsymbol{e}_{i}}\right] / 2$, we can easily derive that $\tilde{\boldsymbol{e}}_{i} \sim \mathcal{N}\left(\mathbf{0}, \widetilde{\boldsymbol{\Sigma}}_{\boldsymbol{e}_{i}}\right)$ utilizing the fact that $\left|d_{i}\right|=1$ and $\boldsymbol{e}_{i} \sim \mathcal{C N}\left(\mathbf{0}, \boldsymbol{\Sigma}_{\boldsymbol{e}_{i}}\right)$.

\section{Proposed Robust CI PReCoding}

In problem $\mathcal{P} 1$, a user $i$ is assumed to be able to detect the data symbol with a required accuracy when the SINR requirement is satisfied, and vice versa. However, due to the existence of $\tilde{\boldsymbol{e}}_{i}$, constraint $\mathrm{C} 1$ cannot be guaranteed definitely. As a substitution, we require that CI should be satisfied with a probability that is large enough, say greater than a given threshold, i.e.:

$$
p_{i} \triangleq \operatorname{Pr}\{\mathrm{C} 1\} \geq \eta_{i}, \forall i \in \mathcal{N}
$$

where $\eta_{i}\left(0.5 \leq \eta_{i} \leq 1\right)$ is the probability required by user $i$. Problem $\mathcal{P} 1$ is then transformed into the following stochastic robust CIPM (SR-CIPM), whose objective is to minimize the total transmit power while guaranteeing the CI constraint with a certain probability for each user:

$$
\begin{aligned}
\mathcal{P} 2(\text { SR-CIPM): } & \min _{\tilde{\boldsymbol{x}}}\|\tilde{\boldsymbol{x}}\|^{2} \\
\text { s.t. }(\mathrm{C} 2): & p_{i} \geq \eta_{i}, \forall i \in \mathcal{N} .
\end{aligned}
$$

Applying the definition of outage probability, if C2 holds, the outage probability of the $i$ th user satisfies:

$$
\bar{p}_{i} \triangleq \operatorname{Pr}\{\operatorname{not} \mathrm{C} 1\}=1-p_{i} \leq 1-\eta_{i} \triangleq v_{i}, \forall i \in \mathcal{N} .
$$

where $v_{i}$ denotes the outage tolerance, i.e. the maximum allowed outage probability, of user $i$.

Since C2 is a probabilistic constraint that is intractable, problem $\mathcal{P} 2$ is not ready to be solved. In the following, we 
transform the problem into a tractable convex optimization problem by properly handling the constraints.

For constraint $\mathrm{C} 1$, we have the following equivalent transformations for $\forall i \in \mathcal{N}$ :

$$
\begin{aligned}
&\left(\tilde{\boldsymbol{h}}_{i}+\tilde{\boldsymbol{e}}_{i}\right)^{\mathrm{T}} \boldsymbol{B} \tilde{\boldsymbol{x}} \mid / \tan \theta \leq\left(\tilde{\boldsymbol{h}}_{i}+\tilde{\boldsymbol{e}}_{i}\right)^{\mathrm{T}} \boldsymbol{A} \tilde{\boldsymbol{x}}-\sqrt{\hat{\gamma}_{i}} \sigma_{z_{i}} \\
& \Leftrightarrow \quad\left\{\begin{array}{l}
\left(\tilde{\boldsymbol{h}}_{i}+\tilde{\boldsymbol{e}}_{i}\right)^{\mathrm{T}} \boldsymbol{B} \tilde{\boldsymbol{x}} / \tan \theta \leq\left(\tilde{\boldsymbol{h}}_{i}+\tilde{\boldsymbol{e}}_{i}\right)^{\mathrm{T}} \boldsymbol{A} \tilde{\boldsymbol{x}}-\sqrt{\hat{\gamma}_{i}} \sigma_{z_{i}} \\
\left(\tilde{\boldsymbol{h}}_{i}+\tilde{\boldsymbol{e}}_{i}\right)^{\mathrm{T}} \boldsymbol{B} \tilde{\boldsymbol{x}} / \tan \theta \geq-\left(\tilde{\boldsymbol{h}}_{i}+\tilde{\boldsymbol{e}}_{i}\right)^{\mathrm{T}} \boldsymbol{A} \tilde{\boldsymbol{x}}+\sqrt{\hat{\gamma}_{i}} \sigma_{z_{i}}
\end{array}\right. \\
& \Leftrightarrow \quad\left\{\begin{array}{l}
\tilde{\boldsymbol{x}}^{\mathrm{T}} \boldsymbol{C} \tilde{\boldsymbol{h}}_{i}+\tilde{\boldsymbol{x}}^{\mathrm{T}} \boldsymbol{C} \tilde{\boldsymbol{e}}_{i}-\sqrt{\hat{\gamma}_{i}} \sigma_{z_{i}} \geq 0 \\
\tilde{\boldsymbol{x}}^{\mathrm{T}} \boldsymbol{D} \tilde{\boldsymbol{h}}_{i}+\tilde{\boldsymbol{x}}^{\mathrm{T}} \boldsymbol{D} \tilde{\boldsymbol{e}}_{i}-\sqrt{\hat{\gamma}_{i}} \sigma_{z_{i}} \geq 0
\end{array}\right.
\end{aligned}
$$

where $\boldsymbol{C}=\left(\boldsymbol{A}-\frac{\boldsymbol{B}}{\tan \theta}\right)^{\mathrm{T}}$ and $\boldsymbol{D}=\left(\boldsymbol{A}+\frac{\boldsymbol{B}}{\tan \theta}\right)^{\mathrm{T}}$. Defining two sets $\mathcal{A}_{i}=\left\{\tilde{\boldsymbol{e}}_{i} \in \mathcal{R}^{2 N \times 1} \mid \tilde{\boldsymbol{x}}^{\mathrm{T}} \boldsymbol{C} \tilde{\boldsymbol{h}}_{i}+\tilde{\boldsymbol{x}}^{\mathrm{T}} \boldsymbol{C} \tilde{\boldsymbol{e}}_{i}-\sqrt{\hat{\gamma}_{i}} \sigma_{z_{i}} \geq 0\right\}$ and $\mathcal{B}_{i}=\left\{\tilde{\boldsymbol{e}}_{i} \in \mathcal{R}^{2 N \times 1} \mid \tilde{\boldsymbol{x}}^{\mathrm{T}} \boldsymbol{D} \tilde{\boldsymbol{h}}_{i}+\tilde{\boldsymbol{x}}^{\mathrm{T}} \boldsymbol{D} \tilde{\boldsymbol{e}}_{i}-\sqrt{\hat{\gamma}_{i}} \sigma_{z_{i}} \geq 0\right\}$, the probability of CI under any given $\tilde{\boldsymbol{x}}$ can then be rewritten and lower bounded as

$$
\begin{aligned}
p_{i}(\tilde{\boldsymbol{x}}) & =\operatorname{Pr}\left\{\tilde{\boldsymbol{e}}_{i} \in\left(\mathcal{A}_{i} \cap \mathcal{B}_{i}\right)\right\} \\
& \geq 1-\operatorname{Pr}\left\{\tilde{\boldsymbol{e}}_{i} \in \overline{\mathcal{A}}_{i}\right\}-\operatorname{Pr}\left\{\tilde{\boldsymbol{e}}_{i} \in \overline{\mathcal{B}}_{i}\right\} .
\end{aligned}
$$

where $\overline{\mathcal{A}}_{i}$ and $\overline{\mathcal{B}}_{i}$ are the complementary sets of $\mathcal{A}_{i}$ and $\mathcal{B}_{i}$, respectively, and the equality holds when $\overline{\mathcal{A}}_{i} \cap \overline{\mathcal{B}}_{i}=\Phi$.

Let $\boldsymbol{\omega}=\boldsymbol{C}^{\mathrm{T}} \tilde{\boldsymbol{x}}$ and $b=\tilde{\boldsymbol{x}}^{\mathrm{T}} \boldsymbol{C} \tilde{\boldsymbol{h}}_{i}-\sqrt{\hat{\gamma}_{i}} \sigma_{z_{i}}$, we have the following transformation:

$$
\operatorname{Pr}\left\{\tilde{\boldsymbol{e}}_{i} \in \overline{\mathcal{A}}_{i}\right\}=\operatorname{Pr}\left\{\boldsymbol{\omega}^{\mathrm{T}} \tilde{\boldsymbol{e}}_{i}<-b\right\} .
$$

Defining $u=\boldsymbol{\omega}^{\mathrm{T}} \tilde{\boldsymbol{e}}_{i}$ and we obtain that $u$ is the weighted sum of $2 N$ independent Gaussian random variables for which we have $u \sim \mathcal{N}\left(0,\left\|\tilde{\boldsymbol{\Sigma}}_{\boldsymbol{e}}^{\frac{1}{2}} \boldsymbol{\omega}\right\|^{2}\right)$. Subsequently, we can calculate $\operatorname{Pr}\left\{\tilde{\boldsymbol{e}}_{i} \in \overline{\mathcal{A}}_{i}\right\}$ as

$$
\begin{aligned}
\operatorname{Pr}\left\{\tilde{\boldsymbol{e}}_{i} \in \overline{\mathcal{A}}_{i}\right\} & =\operatorname{Pr}\{u<-b\} \\
& =\frac{1}{2}\left(1+\operatorname{erf}\left(\frac{-b}{\sqrt{2}\left\|\tilde{\boldsymbol{\Sigma}}_{\boldsymbol{e}}^{\frac{1}{2}} \boldsymbol{\omega}\right\|}\right)\right) \\
& =\frac{1}{2}\left(1-\operatorname{erf}\left(\frac{\tilde{\boldsymbol{x}}^{\mathrm{T}} \boldsymbol{C} \tilde{\boldsymbol{h}}_{i}-\sqrt{\hat{\gamma}_{i}} \sigma_{z_{i}}}{\sqrt{2}\left\|\tilde{\boldsymbol{\Sigma}}_{\boldsymbol{e}}^{\frac{1}{2}} \boldsymbol{C}^{\mathrm{T}} \tilde{\boldsymbol{x}}\right\|}\right)\right)(11)
\end{aligned}
$$

Following the same way, we can also verify that

$$
\operatorname{Pr}\left\{\tilde{\boldsymbol{e}}_{i} \in \overline{\mathcal{B}}_{i}\right\}=\frac{1}{2}\left(1-\operatorname{erf}\left(\frac{\tilde{\boldsymbol{x}}^{\mathrm{T}} \boldsymbol{D} \tilde{\boldsymbol{h}}_{i}-\sqrt{\hat{\gamma}_{i}} \sigma_{z_{i}}}{\sqrt{2}\left\|\tilde{\boldsymbol{\Sigma}}_{\boldsymbol{e}}^{\frac{1}{2}} \boldsymbol{D}^{\mathrm{T}} \tilde{\boldsymbol{x}}\right\|}\right)\right) .
$$

Let $\mu_{i, 1}(\tilde{\boldsymbol{x}})=\frac{\tilde{\boldsymbol{x}}^{\mathrm{T}} \boldsymbol{C} \tilde{\boldsymbol{h}}_{i}-\sqrt{\hat{\gamma}_{i}} \sigma_{z_{i}}}{\sqrt{2}\left\|\tilde{\boldsymbol{\Sigma}}_{\boldsymbol{e}}^{\frac{1}{2}} \boldsymbol{C}^{\mathrm{T}} \tilde{\boldsymbol{x}}\right\|}$ and $\mu_{i, 2}(\tilde{\boldsymbol{x}})=\frac{\tilde{\boldsymbol{x}}^{\mathrm{T}} \boldsymbol{D} \tilde{\boldsymbol{h}}_{i}-\sqrt{\hat{\gamma}_{i}} \sigma_{z_{i}}}{\sqrt{2}\left\|\tilde{\boldsymbol{\Sigma}}_{\boldsymbol{e}}^{\frac{1}{2}} \boldsymbol{D}^{\mathrm{T}} \tilde{\boldsymbol{x}}\right\|}$, (9) can then be rewritten as

$$
p_{i}(\tilde{\boldsymbol{x}}) \geq \frac{1}{2}\left(\operatorname{erf}\left(\mu_{i, 1}(\tilde{\boldsymbol{x}})\right)+\operatorname{erf}\left(\mu_{i, 2}(\tilde{\boldsymbol{x}})\right)\right) \triangleq g\left(\boldsymbol{\mu}_{i}(\tilde{\boldsymbol{x}})\right),
$$

where $\boldsymbol{\mu}_{i}(\tilde{\boldsymbol{x}})=\left(\mu_{i, 1}(\tilde{\boldsymbol{x}}), \mu_{i, 2}(\tilde{\boldsymbol{x}})\right) \in \mathcal{R}^{2}$. (13) indicates that the probability of formulating CI at user $i$ with the given transmit vector $\tilde{\boldsymbol{x}}$ is lower bounded by $g\left(\boldsymbol{\mu}_{i}(\tilde{\boldsymbol{x}})\right)$. This stimulate us to formulate the following problem as a substitute for $\mathcal{P} 2$

$$
\begin{aligned}
\mathcal{P} 3: & \min _{\tilde{\boldsymbol{x}}}\|\tilde{\boldsymbol{x}}\|^{2} \\
\text { s.t. }(\mathrm{C} 3): & g\left(\boldsymbol{\mu}_{i}(\tilde{\boldsymbol{x}})\right) \geq \eta_{i}, \forall i \in \mathcal{N} .
\end{aligned}
$$

However, $\mathcal{P} 3$ is still non-convex. Therefore, further transformation is needed to solve the problem.

Since $\operatorname{erf}(\cdot)$ is a monotonically increasing function, (14b) can be equivalently transformed into the following constraints:

$$
\left\{\begin{array}{l}
\boldsymbol{\mu}_{i}(\tilde{\boldsymbol{x}}) \succeq \boldsymbol{\lambda}_{i} \\
g\left(\boldsymbol{\lambda}_{i}\right)=\eta_{i}
\end{array}\right.
$$

where $\boldsymbol{\lambda}_{i}=\left(\lambda_{i, 1}, \lambda_{i, 2}\right) \in \mathcal{R}^{2}$ is an introduced auxiliary variable. Recalling $\boldsymbol{\mu}_{i}(\tilde{\boldsymbol{x}})$ 's definition, $\boldsymbol{\mu}_{i}(\tilde{\boldsymbol{x}}) \succeq \boldsymbol{\lambda}_{i}$ is equivalent to the following inequalities,

$$
\begin{aligned}
& \sqrt{2} \lambda_{i, 1}\left\|\widetilde{\boldsymbol{\Sigma}}_{\boldsymbol{e}_{i}}^{1 / 2}\left(\boldsymbol{A}-\frac{\boldsymbol{B}}{\tan \theta}\right) \tilde{\boldsymbol{x}}\right\| \leq \tilde{\boldsymbol{h}}_{i}^{\mathrm{T}}\left(\boldsymbol{A}-\frac{\boldsymbol{B}}{\tan \theta}\right) \tilde{\boldsymbol{x}}-\sqrt{\hat{\gamma}_{i}} \sigma_{z_{i}}, \\
& \sqrt{2} \lambda_{i, 2}\left\|\widetilde{\boldsymbol{\Sigma}}_{\boldsymbol{e}_{i}}^{1 / 2}\left(\boldsymbol{A}+\frac{\boldsymbol{B}}{\tan \theta}\right) \tilde{\boldsymbol{x}}\right\| \leq \tilde{\boldsymbol{h}}_{i}^{\mathrm{T}}\left(\boldsymbol{A}+\frac{\boldsymbol{B}}{\tan \theta}\right) \tilde{\boldsymbol{x}}-\sqrt{\hat{\gamma}_{i}} \sigma_{z_{i}} .
\end{aligned}
$$

where each inequality defines a convex quadratic cone.

Define $\Psi_{i}=\left\{\boldsymbol{\mu} \in \mathcal{R}^{2} \mid g(\boldsymbol{\mu}) \geq \eta_{i}\right\}$ and $\mathcal{S}_{i}\left(\boldsymbol{\lambda}_{i}\right)=\{\boldsymbol{\mu} \in$ $\left.\mathcal{R}^{2} \mid \boldsymbol{\mu} \succeq \boldsymbol{\lambda}_{i}\right\}$, from (15) we can derive that

$$
\Psi_{i}=\underset{\bar{\lambda}_{i} \in \mathcal{C}\left(\eta_{i}\right)}{\cup} \mathcal{S}_{i}\left(\overline{\boldsymbol{\lambda}}_{i}\right)
$$

where $\mathcal{C}\left(\eta_{i}\right)=\left\{\boldsymbol{t} \in \mathcal{R}^{2} \mid g(\boldsymbol{t})=\eta_{i}\right\}$. In other words, for any $\overline{\boldsymbol{\lambda}}_{i} \in \mathcal{C}\left(\eta_{i}\right), \mathcal{S}_{i}\left(\overline{\boldsymbol{\lambda}}_{i}\right)$ defines a subset of $\Psi_{i}$. For convenience, in the following we use $\overline{\boldsymbol{\lambda}}_{i}$ to denote any vector in $\mathcal{C}\left(\eta_{i}\right)$, distinguishing it from $\boldsymbol{\lambda}_{i}$ which denotes a vector in $\mathcal{R}^{2}$.

Using the definition of $\Psi_{i}$, it is straight to verify that a vector $\tilde{\boldsymbol{x}} \in \mathcal{R}^{2 M}$ is feasible to $\mathcal{P} 3$ if and only if $\boldsymbol{\mu}_{i}(\tilde{\boldsymbol{x}}) \in \Psi_{i}$. Therefore, $\boldsymbol{\mu}_{i}^{-1}\left(\Psi_{i}\right)$ actually defines the feasible set for $\mathcal{P} 3$ and similarly $\boldsymbol{\mu}_{i}^{-1}\left(\mathcal{S}_{i}\left(\overline{\boldsymbol{\lambda}}_{i}\right)\right)$ defines a sub-feasible set. This stimulates us to replace C3 with (16) which gives us the following problem:

$$
\begin{aligned}
& \mathcal{P} 4: \quad \min _{\tilde{\boldsymbol{x}}}\|\tilde{\boldsymbol{x}}\|^{2} \\
& \text { s.t. (C4): }(16), \forall i \in \mathcal{N} \text {. }
\end{aligned}
$$

Since both objective function and constraints are convex, $\mathcal{P} 4$ is a convex problem that can be efficiently solved using offthe-shelf optimization tools such as CVX. Besides, it is worthy of noticing that the transformation from $\mathcal{P} 3$ to $\mathcal{P} 4$ is actually using $\mathcal{S}_{i}\left(\boldsymbol{\lambda}_{i}\right)$ to replace $\Psi_{i}$. Therefore, how well $\mathcal{S}_{i}\left(\boldsymbol{\lambda}_{i}\right)$ approximates $\Psi_{i}$ will decide how well the transformed problem $\mathcal{P} 4$ approximate the original problem. Besides, we will show that by properly choosing $\boldsymbol{\lambda}_{i}$ 's for each user in $\mathcal{P} 4$, we can create both tightened approximations, with $\mathcal{S}_{i}\left(\boldsymbol{\lambda}_{i}\right) \subset \Psi_{i}$, and relaxed approximations, with $\Psi_{i} \subset \mathcal{S}_{i}\left(\boldsymbol{\lambda}_{i}\right)$, for the constraint $\mathrm{C} 3$ using $\mathrm{C} 4$. The corresponding optimum values of $\mathcal{P} 4$ are then the upper and lower bounds of $\mathcal{P} 3$, respectively.

Considering that $\mathcal{S}_{i}\left(\overline{\boldsymbol{\lambda}}_{i}\right)$ is a subset of $\Psi_{i}$, this motivates us to fix $\boldsymbol{\lambda}_{i}=\overline{\boldsymbol{\lambda}}_{i}$ which yields an upper-bound then according to the above statement. However, in order to find the lowest upper-bound through $\mathcal{P} 4, \bar{\lambda}_{i}$ should be chosen such that the corresponding set $\mathcal{S}_{i}\left(\overline{\boldsymbol{\lambda}}_{i}\right)$ would be the largest subset of $\Psi_{i}$ among all $\mathcal{S}_{i}\left(\overline{\boldsymbol{\lambda}}_{i}\right)$ 's. As $\mathcal{S}_{i}\left(\overline{\boldsymbol{\lambda}}_{i}\right)$ is a cone, the size of it can be 
measured by the area it covers. Subsequently, the optimal $\bar{\lambda}_{i}^{*}$ can be chosen through the following optimization,

$$
\begin{aligned}
\overline{\boldsymbol{\lambda}}_{i}^{*} & =\arg \max _{\overline{\boldsymbol{\lambda}}_{i} \in \mathcal{C}\left(\eta_{i}\right)} S\left(\mathcal{S}_{i}\left(\overline{\boldsymbol{\lambda}}_{i}\right)\right) \\
& =\arg \max _{g\left(\overline{\boldsymbol{\lambda}}_{i}\right)=\eta_{i}}\left(\infty-\bar{\lambda}_{i, 1}\right)\left(\infty-\bar{\lambda}_{i, 2}\right) \\
& =\arg \min _{g(\overline{\boldsymbol{\lambda}})=\eta_{i}}\left(\bar{\lambda}_{i, 1}+\bar{\lambda}_{i, 2}\right)
\end{aligned}
$$

where $S\left(\mathcal{S}_{i}\left(\overline{\boldsymbol{\lambda}}_{i}\right)\right)$ is the area that $\mathcal{S}_{i}\left(\overline{\boldsymbol{\lambda}}_{i}\right)$ covers. Since $g\left(\overline{\boldsymbol{\lambda}}_{i}\right)$ is a symmetric function with respect to the two components $\bar{\lambda}_{i, 1}$ and $\bar{\lambda}_{i, 2}$, i.e. $g\left(\left(\bar{\lambda}_{i, 1}, \bar{\lambda}_{i, 2}\right)\right)=g\left(\left(\bar{\lambda}_{i, 2}, \bar{\lambda}_{i, 1}\right)\right)$, it does not change the result of (19) if we assume $\bar{\lambda}_{i, 2} \geq \bar{\lambda}_{i, 1}$. Therefore, it causes no loss of generality if we let $\bar{\lambda}_{i}=$ $\left(\operatorname{erf}^{-1}(t), \operatorname{erf}^{-1}\left(2 \eta_{i}-t\right)\right)$ where $\eta_{i} \geq t \geq 2 \eta_{i}-1 \geq 0$. Then we have

$$
\begin{aligned}
\min _{g\left(\overline{\boldsymbol{\lambda}}_{i}\right)=\eta_{i}}\left(\bar{\lambda}_{i, 1}+\bar{\lambda}_{i, 2}\right) & =\min _{\eta_{i} \geq t \geq 2 \eta_{i}-1}\left(\operatorname{erf}^{-1}(t)+\operatorname{erf}^{-1}\left(2 \eta_{i}-t\right)\right) \\
& \geq 2 \operatorname{erf}^{-1}\left(\eta_{i}\right),
\end{aligned}
$$

where (20) is derived according to Jensen's inequality since $\operatorname{erf}^{-1}(t)$ is convex when $t \geq 0$ and the equality holds when $t=\eta_{i}$. We can then derive that $\overline{\boldsymbol{\lambda}}_{i}^{*}=\left(\operatorname{erf}^{-1}\left(\eta_{i}\right), \operatorname{erf}^{-1}\left(\eta_{i}\right)\right)$. Conclusively, by setting $\boldsymbol{\lambda}_{i}=\overline{\boldsymbol{\lambda}}_{i}^{*}$, the lowest upper-bound for the optimal transmit power can be obtained through $\mathcal{P} 4$.

Furthermore, for $\forall \overline{\boldsymbol{\lambda}}_{i} \in \mathcal{C}\left(\eta_{i}\right)$ we also have

$$
\begin{aligned}
\bar{\lambda}_{i} & \left.\succeq\left(\operatorname{erf}^{-1}(t), \operatorname{erf}^{-1}(t)\right)\right|_{\eta_{i} \geq t \geq 2 \eta_{i}-1} \\
& \succeq\left(\operatorname{erf}^{-1}\left(2 \eta_{i}-1\right), \operatorname{erf}^{-1}\left(2 \eta_{i}-1\right)\right) \triangleq \boldsymbol{\lambda}_{i}^{l},
\end{aligned}
$$

which indicates that $\mathcal{S}_{i}\left(\overline{\boldsymbol{\lambda}}_{i}^{*}\right) \subset \mathcal{S}_{i}\left(\boldsymbol{\lambda}_{i}^{l}\right)$ for $\forall \overline{\boldsymbol{\lambda}}_{i}$.Therefore,

$$
\mathcal{S}_{i}\left(\overline{\boldsymbol{\lambda}}_{i}^{*}\right) \subset \Psi_{i} \subset \mathcal{S}_{i}\left(\boldsymbol{\lambda}_{i}^{l}\right) .
$$

Thus, $\mathcal{P} 4$ becomes a relaxed problem of $\mathcal{P} 3$ if we let $\boldsymbol{\lambda}_{i}=\boldsymbol{\lambda}_{i}^{l}$ and its optimal value is then a lower bound of the optimal transmit power. Moreover, it is easy to show that for $\forall \boldsymbol{\lambda}_{i}^{\prime}$ satisfying $\Psi_{i} \subset \mathcal{S}_{i}\left(\boldsymbol{\lambda}_{i}^{\prime}\right)$, we always have $\boldsymbol{\lambda}_{i}^{l} \succeq \boldsymbol{\lambda}_{i}^{\prime}$. In other words, $\mathcal{S}_{i}\left(\boldsymbol{\lambda}_{i}^{l}\right)$ is the smallest set that contain $\Psi$ among all $\mathcal{S}_{i}\left(\boldsymbol{\lambda}_{i}\right)$ 's. The proof is directive: suppose there exist $\boldsymbol{\lambda}_{i}^{\prime}$ with $\boldsymbol{\lambda}_{i, 1}^{\prime} \geq \operatorname{erf}^{-1}\left(2 \eta_{i}-1\right)$ but satisfying $\Psi_{i} \subset \mathcal{S}_{i}\left(\boldsymbol{\lambda}_{i}^{\prime}\right)$. It is easy to show that the point $\overline{\boldsymbol{\lambda}}_{i}^{\prime}=\left(\operatorname{erf}^{-1}\left(2 \eta_{i}-1\right), \infty\right)$ satisfies $g\left(\overline{\boldsymbol{\lambda}}_{i}^{\prime}\right)=\eta_{i}$ and thus $\overline{\boldsymbol{\lambda}}_{i}^{\prime} \in \Psi$. However, $\overline{\boldsymbol{\lambda}}_{i}^{\prime} \notin \mathcal{S}_{i}\left(\boldsymbol{\lambda}_{i}^{\prime}\right)$ which is contradict with our assumption that $\Psi_{i} \subset \mathcal{S}_{i}\left(\boldsymbol{\lambda}_{i}^{\prime}\right)$ and thus proves the above analysis. Therefore, by setting $\boldsymbol{\lambda}_{i}=\boldsymbol{\lambda}_{i}^{l}$, we can obtain the best lower bound through solving $\mathcal{P} 4$.

As an illustrative example, we depict the contour line of $g(\boldsymbol{\mu})$ as well as the above defined set when $\eta_{i}=0.9$ in Fig. 1, which shows straightly the relations of $\mathcal{S}_{i}\left(\bar{\lambda}_{i}^{*}\right), \Psi_{i}$ and $\mathcal{S}_{i}\left(\boldsymbol{\lambda}_{i}^{l}\right)$. We can also see from the figure that the larger $\eta_{i}$ is, the better $\mathcal{S}_{i}\left(\overline{\boldsymbol{\lambda}}_{i}^{*}\right)$ and $\mathcal{S}_{i}\left(\boldsymbol{\lambda}_{i}^{l}\right)$ approximate $\Psi_{i}$. Especially, when $\eta_{i} \rightarrow 1$ we have $\overline{\boldsymbol{\lambda}}_{i}^{*}=\boldsymbol{\lambda}_{i}^{l}$ and $\mathcal{S}_{i}\left(\overline{\boldsymbol{\lambda}}_{i}^{*}\right)=\Psi_{i}=\mathcal{S}_{i}\left(\boldsymbol{\lambda}_{i}^{l}\right)$.

\section{Numerical Results}

The performances of the proposed SR-CIPM are investigated through Monte Carlo simulations. In each plot, 8PSK modulation is considered with $M=6$ and $N=4$. Block Rayleigh fading channel are assumed for all channels with $\boldsymbol{h}_{i} \sim \mathcal{C N}\left(\mathbf{0}, \boldsymbol{I}_{M}\right)$ and symmetric assumptions among all users

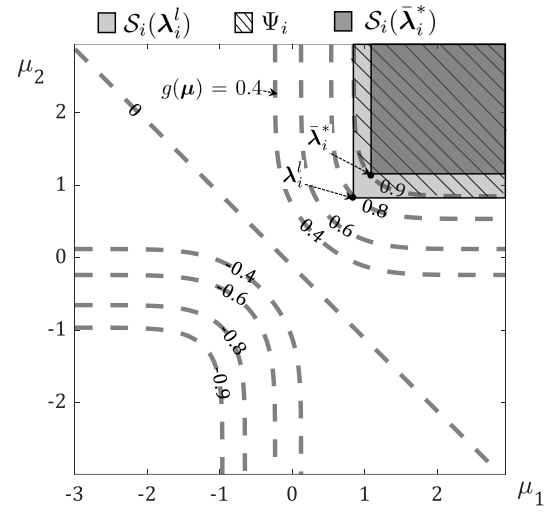

Fig. 1. The contour plot of $g(\boldsymbol{\mu})$ (dashed line) with set $\Psi_{i}, \mathcal{S}_{i}\left(\overline{\boldsymbol{\lambda}}_{i}^{*}\right)$ and $\mathcal{S}_{i}\left(\bar{\lambda}_{i}^{l}\right)$ for $\eta_{i}=0.9$.

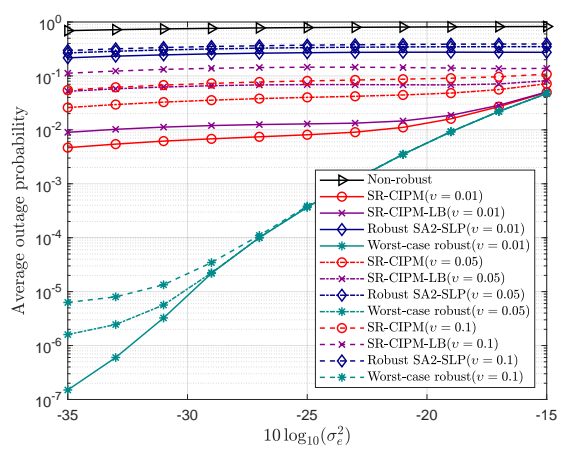

Fig. 2. Probability of outage v.s. channel error variances, $\gamma_{t h}=15 \mathrm{~dB}$.

with $\sigma_{z_{i}}^{2}=1, \hat{\gamma}_{i}=\gamma_{t h}=15 \mathrm{~dB}, v_{i}=v, \eta_{i}=1-v$ and $\boldsymbol{\Sigma}_{\boldsymbol{e}_{i}}=\sigma_{e}^{2} \boldsymbol{I}_{M}$ for $\forall i \in \mathcal{N}$. For clarity, the following abbreviations are used through the section:

- Non-robust: the CI precoding scheme which does not consider channel errors;

- Worst-case robust: the worst-case robust CI precoding in [5];

- Robust SA2-SLP: the robust SA2-SLP scheme in [21];

- SR-CIPM: the solution to $\mathcal{P} 4$ with $\bar{\lambda}=\bar{\lambda}_{i}^{*}$;

- SR-CIPM-LB: the solution to $\mathcal{P} 4$ with $\bar{\lambda}=\lambda_{i}^{l}$ which acts as a lower bound of the original stochastic CIPM.

For the worst-case robust scheme, $\mathrm{CI}$ is supposed to be absolutely guaranteed when channel error is bounded by a ball $\left\{\boldsymbol{e}_{i}\|\| \boldsymbol{e}_{i}\left\|_{\bar{\sigma}_{e}}\right\|^{2} \leq \Phi_{2 M}^{-1}\left(\eta_{i}\right)\right\}$ where $\Phi_{2 M}^{-1}(\cdot)$ is the inverse of the Chi-siquare cumulative distribution function with $2 \mathrm{M}$ degrees of freedom. This assumption ensures that the probability of $\boldsymbol{e}_{i}$ being in the ball is exactly $\eta_{i}$. A maximum transmit power $p_{\max }=\left(25+\gamma_{t h}\right) \mathrm{dBw}$ is assumed to avoid unacceptable high transmit powers and the non-robust CIPM precoder[5] with power normalized to $p_{\max }$ would be used when the robust schemes are infeasible. The performances of these schemes under different $\sigma_{e}$ 's and $v$ 's are first simulated assuming $\gamma_{t h}=15 \mathrm{~dB}$ and illustrated in Fig.2, to Fig. 5 .

In Fig. 2, we show the average outage probability versus $\sigma_{e}$ in the logarithm scale to investigate their ability to satisfy users' QoS requirements. We can see that the outage probabilities of the non-robust scheme are much larger than the allowed 
tolerances and deteriorate as $\sigma_{e}$ increases. This rationalizes the necessity of designing robust CI schemes to combat the unavoidable channel uncertainties in real applications. And as might be expected, the robust SA2-SLP method failed in meeting the requirements either. For example, at $\sigma_{e}=-35 \mathrm{~dB}$, its outage probabilities are $0.30,0.26$ and 0.22 for $v=0.1$, 0.05 and 0.01 , respectively, which are far above the allowed tolerances. This is because its transformed constraints can not accurately reflect users' outage requirements, which can also be observed in its slow reaction to outage requirements variations. As $v$ decreases rapidly from 0.1 to 0.01 , its outage probabilities remain almost the same. On the other hand, our proposed schemes work well in most scenarios. In all scenarios, the outage probabilities of SR-CIPM are slightly below the allowed tolerances, while that of SR-CIPM-LB are slightly above them, which agrees with our conclusion on the upper and lower bound statements. However, when channel uncertainty becomes too severe, say when $\sigma_{e}$ exceeds $-21 \mathrm{~dB}$ (for $v=0.01$ ), $-17 \mathrm{~dB}$ (for $v=0.05$ ) and $-15 \mathrm{~dB}$ (for $v=0.1$ ), SR-CIPM either failed to guarantee the outage performances, which is due to the rapid degradation of the feasibility rate as shown in Fig. 4. Nevertheless, its performances are still very close to the required values. The worst-case robust scheme yields the lowest outage probabilities, which are far below the allowed tolerances, resulting in unnecessary power consumption.

Fig. 3 illustrates the average transmit power consumption of these schemes. As shown, the proposed SR-CIPM consumes much less transmit power than the worst-case robust. Particularly, the power increasing of SR-CIPM is slower than the worst-case robust scheme, making the gain more prominent in a relatively high CE regime. Moreover, the gain increases when $v$ getting larger, with the maximum gains achieved around $\sigma_{e}=-27 \mathrm{~dB}$ which are about $8 \mathrm{~dB}, 10 \mathrm{~dB}$ and $11 \mathrm{~dB}$ for $v=0.01,0.05$ and 0.1 , respectively. This trend indicates that the proposed scheme is more adaptive to changes of $v$. Please note that the gain is narrowed when $\sigma_{e}$ becomes too large, which is because of the maximum transmit power limitation other than the scheme itself. We can also observe that the power consumption of SR-CIPM is very close to that of SR-CIPM-LB in all cases. Considering the upper and lower bound property of the two schemes, this indicates that our approximating methods do not cause obvious performance loss compared with the potential optimal solution. Besides, their gap becomes smaller when $v$ decreases, verifying our analysis that our approximation becomes more accurate when $\eta \rightarrow 1$. Fig. 3 also shows that robust schemes' transmit power increase as $\sigma_{e}$ increases or $v$ decreases. Fig. 4 shows the feasibility rate of robust schemes. We can see that they all decrease rapidly when $\sigma_{e}$ exceeds specific values and become less feasible as $v$ decreases. However, compared with the worst-case robust scheme, the proposed methods can tolerate much higher channel uncertainties. Besides, SA2-SLP shows a good feasibility performance, which is due to its failure to reflect the outage requirement accurately.

To investigate how different conservatism levels in satisfying the CI constraints affect the users' performance, we also depict the average symbol error rate (SER) of these

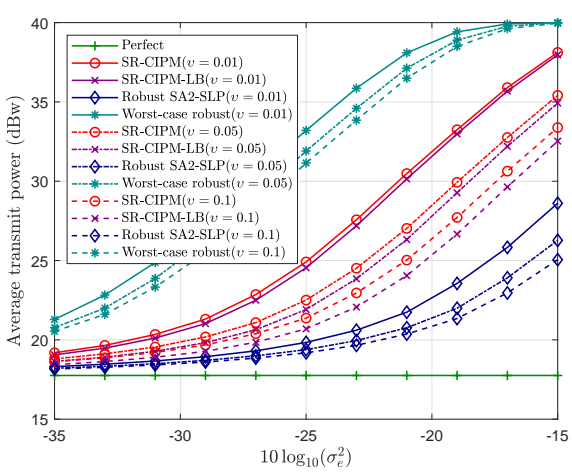

Fig. 3. Transmit power v.s. channel error variances, $\gamma_{t h}=15 \mathrm{~dB}$.

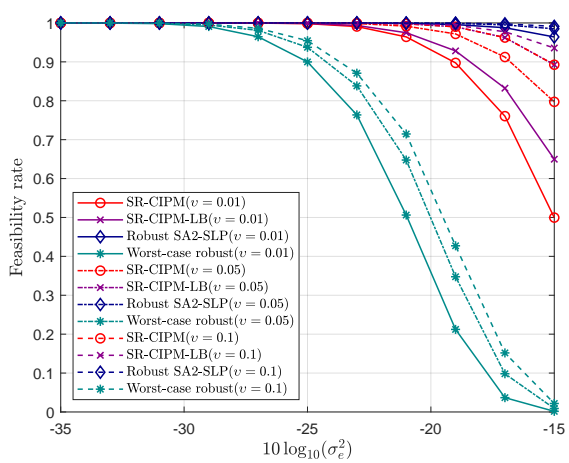

Fig. 4. Feasibility rate v.s. channel error variances, $\gamma_{t h}=15 \mathrm{~dB}$.

schemes in Fig. 5. As shown, the SER performance of the non-robust scheme degrades rapidly as CE increases. While the SER of robust schemes remains below that of CIPM with perfect CSIT until $\sigma_{e}$ exceeds a certain threshold. Considering the high outage probability of SA2-SLP, this indicates that $v$ can be set relatively large when $\sigma_{e}$ is smaller than the threshold. However, we can also observe that the schemes which yield lower outage probabilities can tolerate higher channel uncertainties. Besides, a trend that the SER firstly decreases and then increases with $\sigma_{e}$ can be seen in SRCIPM, SR-CIPM-LB, and the worst-case robust schemes. The decrease is because more residuals are created to combat the increasing uncertainty. However, at high $\sigma_{e}$ regimes, the maximum power limitations and the decreased feasibility rate begin to take effect, which causes an increase of SER. In all scenarios, the SER of SR-CIPM-LB is slightly above that of SR-CIPM. And the performance gap between the two schemes decreases as $v$ decreases, further qualifying our approximation method and their upper and lower bound property.

Fig. 6 further illustrate the SER performances under different SINR target with $\sigma_{e}=-23 d B$. Apparently, an error floor can be seen at high SINR for both non-robust and robust schemes due to imperfect CSIT. However, the error floor of the proposed schemes and the worst-case robust scheme is lower than that of SA2-SLP and the non-robust scheme, which is due to their ability of achieving lower outage probabilities. Furthermore, from Fig. 5 and Fig. 6, we can also see that as the outage tolerance $v$ decreases, the SER of the proposed schemes also decrease obviously while that of SA2-SLP remains almost the 


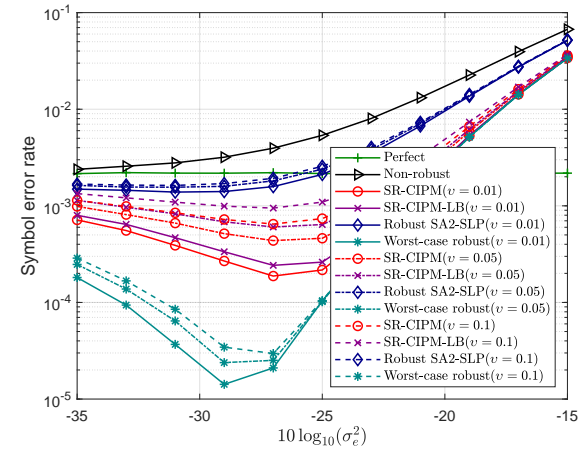

Fig. 5. Bit error rate v.s. channel error variances, $\gamma_{t h}=15 \mathrm{~dB}$.

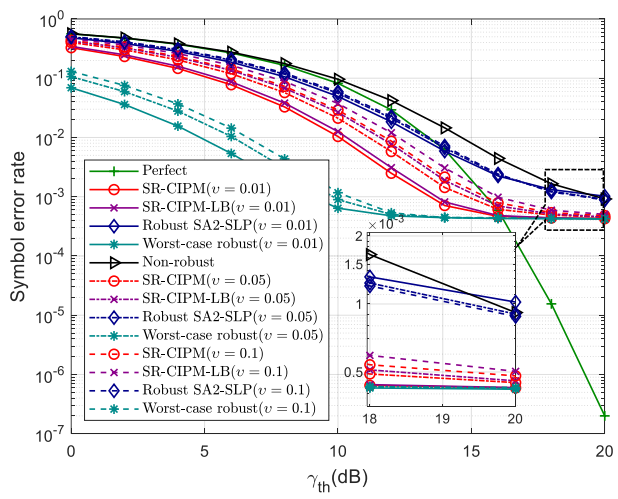

Fig. 6. Bit error rate v.s. target SINR, $\sigma_{e}=-23 d B$.

same. This again implies that the proposed schemes can reflect users' requirements more accurately, which enables the system to change the performance by controlling the value of $v$.

\section{CONCLUSION}

In this paper, we propose a stochastic robust CI precoding for MISO downlink systems with contaminated CSIT. The CIPM problem is formulated into an outage-constrained problem by considering CEs. Based on a detailed analysis of the probabilistic constraints, we transform them into determined and convex constraints through approximations and change the intractable problem into SOC programming. We show that by properly selecting the parameters, the performance loss caused by transforming the constraints can be negligible. Our simulation results also show that the proposed scheme can reliably guarantee the outage requirement with much lower power consumption than the worst-case robust scheme and works more steadily under different channel uncertainty levels.

\section{REFERENCES}

[1] M. Alodeh et al., "Symbol-Level and Multicast Precoding for Multiuser Multiantenna Downlink: A State-of-the-Art, Classification, and Challenges," IEEE Communications Surveys Tutorials, vol. 20, no. 3, pp. 1733-1757, Thirdquarter 2018.

[2] J. Wang and D. P. Palomar, "Worst-Case Robust MIMO Transmission With Imperfect Channel Knowledge," IEEE Transactions on Signal Processing, vol. 57, no. 8, pp. 3086-3100, Aug. 2009.

[3] K. Wang; A. So; T. Chang; W. Ma; C. Chi, "Outage Constrained Robust Transmit Optimization for Multiuser MISO Downlinks: Tractable Approximations by Conic Optimization," IEEE Transactions on Signal Processing, vol. 62, no. 21, pp. 5690-5705, Nov. 2014.
[4] A. Li, D. Spano, J. Krivochiza, S. Domouchtsidis, C. Tsinos, C. Masouros, S. Chatzinotas, Y. Li, B. Vucetic, and B. Ottersten, "A Tutorial on Interference Exploitation via Symbol-Level Precoding: Overview, Stateof-the-Art and Future Directions," IEEE Commun. Surveys and Tutorials, vol. 22, no. 2, pp. 796-839, Secondquarter 2020.

[5] C. Masouros and G. Zheng, "Exploiting Known Interference as Green Signal Power for Downlink Beamforming Optimization," IEEE Trans. Signal Process., vol. 63, no. 14, pp. 3628-3640, Jul. 2015.

[6] C. Masouros, T. Ratnarajah, M. Sellathurai, C. B. Papadias, and A. K. Shukla, "Known interference in the cellular downlink: A performance limiting factor or a source of green signal power?" IEEE Commun. Mag., vol. 51, no. 10, pp. 162-171, Oct. 2013.

[7] G. Zheng, I. Krikidis, C. Masouros, S. Timotheou, D.-A. Toumpakaris, and Z. Ding, "Rethinking the role of interference in wireless networks", IEEE Commun. Mag., vol. 52, no. 11, pp. 152-158, Nov. 2014.

[8] C. Masouros, "Correlation rotation linear precoding for MIMO broadcast communications," IEEE Trans. Signal Process, vol. 59, no. 1, pp. 252-262, Jan. 2011.

[9] C. Masouros and E. Alsusa, "Dynamic linear precoding for the exploitation of known interference in MIMO broadcast systems," IEEE Trans. Wireless Commun., vol. 8, no. 3, pp. 1396-1404, Mar. 2009.

[10] M. Alodeh, S. Chatzinotas, and B. E. Ottersten, "Constructive multiuser interference in symbol level precoding for the MISO downlink channel," IEEE Trans. Signal Process., vol. 63, no. 9, pp. 2239-2252, May 2015.

[11] M. Alodeh, S. Chatzinotas, and B. E. Ottersten, "Energy-efficient symbol-level precoding in multiuser MISO based on relaxed detection region", IEEE Trans. Wireless Commun., vol. 15, no. 5, pp. 3755-3767, May 2016.

[12] M. Alodeh, S. Chatzinotas, and B. E. Ottersten, "Symbol-level multiuser MISO precoding for multi-level adaptive modulation," IEEE Trans. Wireless Commun., vol. 16, no. 8, pp. 5511-5524, Aug. 2017.

[13] A. Li and C. Masouros, "Interference exploitation precoding made practical: Optimal closed-form solutions for PSK modulations," IEEE Trans. Wireless Commun., vol. 17, no. 11, pp. 7661-7676, Nov. 2018.

[14] A. R. Haqiqatnejad, F. Kayhan, and B. E. Ottersten, "Constructive interference for generic constellations," IEEE Signal Process. Lett., vol. 25, no. 4, pp. 586-590, Apr. 2018.

[15] A. Li, C. Masouros, X. Liao, Y. Li, and B. Vucetic, "Multiplexing more data streams in the MU-MISO downlink by interference exploitation precoding," in Proc. IEEE Wireless Commun. Netw. Conf. (WCNC), Seoul, South Korea, 2020, pp. 1-6.

[16] K. L. Law and C. Masouros, "Symbol error rate minimization precoding for interference exploitation," IEEE Trans. Commun., vol. 66, no. 11, pp. 5718-5731, Nov. 2018.

[17] Z. Wei and C. Masouros, "Device-Centric Distributed Antenna Transmission: Secure Precoding and Antenna Selection With Interference Exploitation," IEEE IoT Journal, vol. 7, no. 3, pp. 2293-2308, March 2020.

[18] Z. Wei, C. Masouros, F. Liu, S. Chatzinotas and B. Ottersten, "Energyand Cost-Efficient Physical Layer Security in the Era of IoT: The Role of Interference," IEEE Commun. Mag., vol. 58, no. 4, pp. 81-87, April 2020.

[19] A. R. Haqiqatnejad, F. Kayhan, and B. E. Ottersten, "Power minimizer symbol-level precoding: A closed-form suboptimal solution," IEEE Signal Process. Lett., vol. 25, no. 11, pp. 1730-1734, Nov. 2018.

[20] D. Kwon, H. S. Kang and D. K. Kim, "Robust Interference ExploitationBased Precoding algorithm With Quantized CSIT," IEEE Commun. Lett., vol. 20, no. 4, pp. 780-783, Apr. 2016.

[21] A. Haqiqatnejad, F. Kayhan and B. Ottersten, "Robust SINR-Constrained Symbol-Level Multiuser Precoding With Imperfect Channel Knowledge," IEEE Trans. Signal Process., vol. 68, pp. 1837-1852, 2020.

[22] Z. Wei, C. Masouros, K. Wong and X. Kang, "Multi-Cell Interference Exploitation: Enhancing the Power Efficiency in Cell Coordination," IEEE Transactions on Wireless Communications, vol. 19, no. 1, pp. 547-562, Jan. 2020.

[23] A. Haqiqatnejad, F. Kayhan and B. Ottersten, "Constructive Interference for Generic Constellations," IEEE Signal Process. Lett., vol. 25, no. 4, pp. 586-590, Apr. 2018.

[24] H. A. Fayed and A. F. Atiya, "An Evaluation of the Integral of the Product of the Error Function and the Normal Probability Density with Application to the Bivariate Normal Integral," Mathematics of Computation, vol. 83, no. 285, pp. 235-250, Jan. 2014.

[25] A. Ben-Tal and A. Nemirovski, "Lectures on Modern Convex Optimization: Analysis, Algorithms, and Engineering Applications," Philadelphia: SIAM, 2001. 